CARPATHIAN J. MATH.

Volume 37 (2021), No. 1,

Pages $53-63$
Online version at https : //www. carpathian. cunbm. utcluj.ro/

Print Edition: ISSN 1584 - 2851; Online Edition: ISSN 1843 - 4401

DOI: https://doi.org/10.37193/CJM.2021.01.06

\title{
Ideals in residuated lattices
}

\author{
Dumitru Buşneag, Dana Piciu and Anca-Maria Dina
}

\begin{abstract}
The notion of ideal in residuated lattices is introduced in [Kengne, P. C., Koguep, B. B., Akume, D. and Lele, C., $\mathcal{L}$-fuzzy ideals of residuated lattices, Discuss. Math. Gen. Algebra Appl., 39 (2019), No. 2, 181-201] and [Liu, Y., Qin, Y., Qin, X. and Xu, Y., Ideals and fuzzy ideals in residuated lattices, Int. J. Math. Learn \& Cyber., 8 (2017), 239-253] as a natural generalization of that of ideal in $M V$ algebras (see [Cignoli, R., D'Ottaviano, I. M. L. and Mundici, D., Algebraic Foundations of many-valued Reasoning, Trends in Logic-Studia Logica Library 7, Dordrecht: Kluwer Academic Publishers, 2000] and [Chang, C. C., Algebraic analysis of many-valued logic, Trans. Amer. Math. Soc., 88 (1958), 467-490]). If $A$ is an MV algebra and $I$ is an ideal on $A$ then the binary relation $x \sim_{I} y$ iff $x^{*} \odot y, x \odot y^{*} \in I$, for $x, y \in A$, is a congruence relation on $A$. In this paper we find classes of residuated lattices for which the relation $\sim_{I}$ (defined for MV algebras) is a congruence relation and we give new characterizations for i-ideals and prime i-ideals in residuated lattices. As a generalization of the case of BL algebras (see [Lele, C. and Nganou, J. B., MV-algebras derived from ideals in BL-algebras, Fuzzy Sets and Systems, 218 (2013), 103-113]), we investigate the relationship between i-ideals and deductive systems in residuated lattices.
\end{abstract}

\section{INTRODUCTION}

Residuated lattices have been investigated in [5], [8], [15]. Apart from their logical interest, these lattices have interesting algebraic properties and include two important classes of algebras: BL algebras and MV algebras. The notion of ideal has been introduced in many algebraic structures (lattices, rings and MV algebras). The ideal theory is a very effective tool for studying various algebraic and logical systems.

In modern fuzzy logic theory, residuated lattices and some related algebraic systems, play an important role because they provide an algebraic frameworks to fuzzy logic and fuzzy reasoning. By using the theory of residuated lattices, Pavelka has built up a more generalized logic systems and he has proved the semantical completeness of the Lukasiewicz's axiom system (see [12]). From a logical point of view, various filters and ideals corresponding to various sets of provable formulae.

In rings, MV algebras and lattice implication algebras, the ideal is in the center position. However, in BL algebras, MTL algebras or residuated lattices the focus has been on filters or deductive systems.

By definition, ideals in MV algebras are kernels of homomorphisms ([3]). In residuated lattices the notion of ideal was introduced as a natural generalization of that of ideal in MV algebras and the relation between filters and ideals was discussed (see [9], [10] for BL algebras and [7], [11] for residuated lattices).

It is known the fact that an ideal is a dual of a filter in some special logical algebras (for example, in lattice implication algebras). Unlike in lattice implication algebras, ideals and the dual of filters be quite differently in non regular residuated lattices, see [11].

We recall that if $I$ is an ideal of an MV algebra $A$, then the binary relation $x \sim_{I} y$ iff $x^{*} \odot y, x \odot y^{*} \in I$, for $x, y \in A$, is a congruence relation on $A$. In [11], for an i-ideal $I$

Received: 23.10.2019. In revised form: 02.09.2020. Accepted: 09.09.2020

2010 Mathematics Subject Classification. 03B50, 06A06, 03G05, 08A72, 06D35, 06B20, 03 C05.

Key words and phrases. Residuated lattice, $M V$ algebra, i-ideal, prime $i$-ideal, deductive system.

Corresponding author: Dumitru Buşneag; busneag@central.ucv.ro 
in a residuated lattice $L$ is defined a congruence relation on $L, x \approx_{I} y$ iff $(x \rightarrow y)^{*}$ and $(y \rightarrow x)^{*} \in I$.

In this paper we study the relationships between the binary relations $\sim_{I}$ and $\approx_{I}$ on residuated lattices. Also we find other classes of residuated lattices for which the relation $\sim_{I}$ (defined for MV algebras) is a congruence relation. Thus, we show that if $L$ is a divisible residuated lattice, a Girard monoid or a De Morgan residuated lattice satisfying the identity $(x \rightarrow y)^{* *}=x^{* *} \rightarrow y^{* *}$ then $\sim_{I}$ is a congruence relation on $L$, see Theorem 3.4. Moreover, if $L$ is a divisible residuated lattice and $I \in \mathbf{I}_{i}(\mathbf{L})$, then $\left(L / I, \boxplus,{ }^{*}, I\right)$ is an MV algebra, see Corollary 3.1. The notion of prime i-ideal in residuated lattices coincides with the concept of prime ideal in MV algebras. Then we attempt, to generalize some results about prime ideals in residuated lattices instead MV algebras. Also, we establish the relationship between ideals and filters (deductive systems) in residuated lattices (see Theorems 4.7, 4.8 and Corollary 4.2).

\section{PRELIMinaries}

A residuated lattice ([5], [14], [15]) is an algebra $(L, \vee, \wedge, \odot, \rightarrow, 0,1)$, where $\vee, \wedge, \odot, \rightarrow$ are binary operations on $L$ and $0,1 \in L$ such that:

$\left(R L_{1}\right)(L, \vee, \wedge, 0,1)$ is a bounded lattice (with partial order denoted by $\leq$ );

$\left(R L_{2}\right)(L, \odot, 1)$ is a commutative monoid;

$\left(R L_{3}\right)$ For $x, y, z \in L, x \odot z \leq y$ iff $z \leq x \rightarrow y$ (the law of residuation).

In a residuated lattice $L$ we consider the following identities:

(div) $x \odot(x \rightarrow y)=x \wedge y$ (divisibility);

(prel) $(x \rightarrow y) \vee(y \rightarrow x)=1$ (prelinearity);

$(D N) x^{* *}=x$ (Double Negation), for every $x, y \in L$.

Definition 2.1. ([3], [6], [14]) A residuated lattice $L$ is called

(i) divisible if $L$ verifies (div);

(ii) an MTL algebra if $L$ verifies (prel);

(iii) a $B L$-algebra if $L$ verifies (div) and (prel);

(iv) a Girard monoid if $L$ verifies $(D N)$;

(v) an MV algebra if it satisfies the additional condition: $(x \rightarrow y) \rightarrow y=(y \rightarrow x) \rightarrow x$, for every $x, y \in L$;

(vi) a De Morgan residuated lattice if it satisfies the identity: $(x \wedge y)^{*}=x^{*} \vee y^{*}$, for every $x, y \in L$. Examples of De Morgan residuated lattices are Boolean algebras, MV algebras, BL algebras, MTL algebras and Girard monoids.

Let $L$ be a residuated lattice. For $x \in L$ and $n \geq 2$ we define $x^{n}=x^{n-1} \odot x, x^{*}=x \rightarrow 0$ and $x^{* *}=\left(x^{*}\right)^{*}$.

For $x, y, z \in L$ we have the following rules of calculus (see [2] and [11]):

$\left(c_{1}\right) 1 \rightarrow x=x$ and $x \leq y$ iff $x \rightarrow y=1$;

$\left(c_{2}\right)$ If $x \leq y$, then $z \odot x \leq z \odot y, z \rightarrow x \leq z \rightarrow y, y \rightarrow z \leq x \rightarrow z$;

$\left(c_{3}\right) x \odot(y \vee z)=(x \odot y) \vee(x \odot z), x \rightarrow(y \wedge z)=(x \rightarrow y) \wedge(x \rightarrow z), x \rightarrow(y \rightarrow z)=$ $(x \odot y) \rightarrow z$

$\left(c_{4}\right) 0^{*}=1,1^{*}=0, x \odot x^{*}=0, x \leq x^{* *}, x^{* * *}=x^{*},(x \vee y)^{*}=x^{*} \wedge y^{*}, x \rightarrow y^{*}=$ $(x \odot y)^{*}=y \rightarrow x^{*}$

$\left(c_{5}\right)\left(x^{* *} \wedge y^{* *}\right)^{* *}=x^{* *} \wedge y^{* *},(x \odot y)^{* *}=x^{* *} \odot y^{* *}$.

For $x, y \in L$, we define $x \boxplus y=\left(x^{*} \odot y^{*}\right)^{*} \stackrel{\left(c_{4}\right)}{=} x^{*} \rightarrow y^{* *}$ and $x \rightsquigarrow y=x^{*} \odot y$.

If $x, y, z \in L$, then we have the following rules of calculus (see [2]):

$\left(c_{6}\right) x, y \leq x \boxplus y, x \boxplus y=y \boxplus x, x \boxplus 0=x^{* *}, x \boxplus x^{*}=1,(x \boxplus y) \boxplus z=x \boxplus(y \boxplus z)$. 


\section{I-IDEALS AND THE BINARY RELATION $\sim_{I}$}

In residuated lattices the notion of ideal (called implicative ideal or $i$-ideal) generalizes the notion of ideal in $M V$ algebras and $B L$ algebras, see [3] and [11]. Unlike in $M V$-algebras, ideals and filters behave quite differently in residuated lattices.

Definition 3.2. ([11]) A nonempty subset $I$ of a residuated lattice $L$ is called implicative ideal (or $i$-ideal) of $L$ if :

$\left(I_{1}\right)$ If $x \leq y$ and $y \in I$, then $x \in I$;

$\left(I_{2}\right)$ If $x, y \in I$, then $x^{*} \rightarrow y \in I$.

Remark 3.1. If $I$ is an i-ideal of $L$, then $0 \in I$ and $x \in I$ iff $x^{* *} \in I$. Also, using $(i), I$ is an i-ideal of $L$ if it satisfies $\left(I_{1}\right)$ and $\left(I_{2}^{\prime}\right):$ If $x, y \in I$, then $x \boxplus y \in I$.

Obviously, every i-ideal is a latticeal ideal, but the converse is not true. If the residuated lattice is an MV algebra, the notion of i-ideal as well as the concept of ideal in MV algebras coincide.

An i-ideal $I$ is called proper if $I \neq L$. We denote by $\mathbf{I}_{i}(\mathbf{L})$ the set of all i-ideals of $L$.

Example 3.1. ([6]) Let $L=\{0, n, a, b, c, d, 1\}$ with $0<n<a<b<c, d<1$, and $c, d$ are incomparable. $L$ becomes a residuated lattice relative to the following operations:

\begin{tabular}{|c|c|c|c|c|c|c|c|c|c|c|c|c|c|c|c|}
\hline$\rightarrow$ & 0 & $n$ & $a$ & $b$ & $c$ & $d$ & 1 & $\odot$ & 0 & $n$ & $a$ & $b$ & $c$ & $d$ & 1 \\
\hline 0 & 1 & 1 & 1 & 1 & 1 & 1 & 1 & 0 & 0 & 0 & 0 & 0 & 0 & 0 & 0 \\
\hline$n$ & $d$ & 1 & 1 & 1 & 1 & 1 & 1 & $n$ & 0 & 0 & 0 & 0 & $n$ & 0 & $n$ \\
\hline$a$ & $n$ & $n$ & 1 & 1 & 1 & 1 & 1 & $a$ & 0 & 0 & $a$ & $a$ & $a$ & $a$ & $a$ \\
\hline$b$ & $n$ & $n$ & $a$ & 1 & 1 & 1 & 1 & $b$ & 0 & 0 & $a$ & $b$ & $b$ & $b$ & $b$ \\
\hline$c$ & 0 & $n$ & $a$ & $d$ & 1 & $d$ & 1 & $c$ & 0 & $n$ & $a$ & $b$ & $c$ & $b$ & $c$ \\
\hline$d$ & $n$ & $n$ & $a$ & $c$ & $c$ & 1 & 1 & $d$ & 0 & 0 & $a$ & $b$ & $b$ & $d$ & $d$ \\
\hline 1 & 0 & $n$ & $a$ & $b$ & $c$ & $d$ & 1 & 1 & 0 & $n$ & $a$ & $b$ & $c$ & $d$ & 1 \\
\hline
\end{tabular}

It is immediate that $\{0, n\}$ is the only proper i-ideal of $L$, so $\mathbf{I}_{i}(\mathbf{L})=\{\{0\},\{0, n\}, L\}$.

Remark 3.2. The residuated lattice from Example 3.1 is not an MV algebra since for example, $(a \rightarrow b) \rightarrow b=b \neq 1=(b \rightarrow a) \rightarrow a$.

Let $L$ be a residuated lattice and $S \subseteq L$ be a nonempty subset of $L$. We denote by $(S]$ the i-ideal of $L$ generated by $S$ (that is, $(S]$ is the least i-ideal of $L$ containing $S$ ).

For $I \in \mathbf{I}_{i}(\mathbf{L})$ and $a \in L$ we denote $I(a)=(I \cup\{a\}]$.

Also, for $x \in L$ and a natural number $n \geq 1$ we denote $n x=x \boxplus \ldots \boxplus x$, where $n$ indicates the number of occurrences of $x$.

Proposition 3.1. ([2]) Let $L$ be a residuated lattice, $S \subseteq L$ a nonempty subset, $a \in L$ and $I \in \mathbf{I}_{i}(\mathbf{L})$. Then:

(i) $(S]=\left\{x \in L: x \leq s_{1} \boxplus \ldots \boxplus s_{n}\right.$, for some $n \geq 1$ and $\left.s_{1}, \ldots, s_{n} \in S\right\}$;

(ii) $I(a)=\{x \in L: x \leq i \boxplus n a$, for some $i \in I$ and $n \geq 1\}$;

(iii) $\left(\mathbf{I}_{i}(\mathbf{L}), \subseteq\right)$ is a complete lattice, where for $I_{1}, I_{2} \in \mathbf{I}_{i}(\mathbf{L}), I_{1} \wedge I_{2}=I_{1} \cap I_{2}$ and $I_{1} \vee I_{2}$ $=\left(I_{1} \cup I_{2}\right]=\left\{x \in L: x \leq i_{1} \boxplus i_{2}\right.$, with $i_{1} \in I_{1}$ and $\left.i_{2} \in I_{2}\right\}$.

Proposition 3.2. In a residuated lattice $L$ we have the following rules of calculus for every $x, y, z \in L:$

$\left(c_{7}\right) x \rightsquigarrow x=0,0 \rightsquigarrow x=x, x \rightsquigarrow 0=0, x \rightsquigarrow 1=x^{*}, 1 \rightsquigarrow x=0, x \rightsquigarrow x^{* *}=0 ;$

(c $\left.c_{8}\right) y \leq x \boxplus z$ iff $x \rightsquigarrow y \leq z^{* *}$ and $x \rightsquigarrow y=0$ iff $y \leq x^{* *}$;

$\left(c_{9}\right)$ If $x \leq y$ then $z \rightsquigarrow x \leq z \rightsquigarrow y$ and $y \rightsquigarrow z \leq x \rightsquigarrow z$;

$\left(c_{10}\right) x \rightsquigarrow y \leq y,(x \rightsquigarrow y) \rightsquigarrow y \leq x^{* *}, x \rightsquigarrow(y \rightsquigarrow z)=y \rightsquigarrow(x \rightsquigarrow z)$; 
$\left(c_{11}\right) x \rightsquigarrow(y \vee z)=(x \rightsquigarrow y) \vee(x \rightsquigarrow z) ;$

$\left(c_{12}\right) \quad(x \boxplus y)^{* *}=x \boxplus y$ and $(n x)^{* *}=n x$, for $n \geq 2$;

$\left(c_{13}\right) x \boxplus\left(y^{* *} \wedge z^{* *}\right)=(x \boxplus y) \wedge(x \boxplus z) ;$

$\left(c_{14}\right) x \wedge(y \boxplus z) \leq\left(x^{* *} \wedge y^{* *}\right) \boxplus\left(x^{* *} \wedge z^{* *}\right) ;$

$\left(c_{15}\right) x \wedge(n y) \leq n\left(x^{* *} \wedge y^{* *}\right)$ and $(m x) \wedge(n y) \leq(m n)\left(x^{* *} \wedge y^{* *}\right)$ for every $m, n \geq 2$.

Proof. $\left(c_{7}\right)$. Obviously.

$\left(c_{8}\right)$. We have $y \leq x \boxplus z$ iff $y \rightarrow(x \boxplus z)=1$ iff $y \rightarrow\left(x^{*} \rightarrow z^{* *}\right)=1$ iff $\left(x^{*} \odot y\right) \rightarrow z^{* *}=1$ iff $x \rightsquigarrow y \leq z^{* *}$. For $z=0, x \rightsquigarrow y=0$ iff $y \leq x^{* *}$.

$\left(c_{9}\right)$. If $x \leq y$ then $z^{*} \odot x \leq z^{*} \odot y$, that is, $z \rightsquigarrow x \leq z \rightsquigarrow y$. Also, from $y^{*} \leq x^{*}$ we deduce that $y^{*} \odot z \leq x^{*} \odot z$, so, $y \rightsquigarrow z \leq x \rightsquigarrow z$.

$\left(c_{10}\right)$. Clearly, $x \rightsquigarrow y=x^{*} \odot y \leq y$ and $(x \rightsquigarrow y) \rightsquigarrow y=\left(x^{*} \odot y\right)^{*} \odot y=y \odot\left(y \rightarrow x^{* *}\right) \leq x^{* *}$. Also, $x \rightsquigarrow(y \rightsquigarrow z)=x^{*} \odot\left(y^{*} \odot z\right)=y^{*} \odot\left(x^{*} \odot z\right)=y \rightsquigarrow(x \rightsquigarrow z)$.

$\left(c_{11}\right) . x \rightsquigarrow(y \vee z)=x^{*} \odot(y \vee z)=\left(x^{*} \odot y\right) \vee\left(x^{*} \odot z\right)=(x \rightsquigarrow y) \vee(x \rightsquigarrow z)$.

$\left(c_{12}\right) .(x \boxplus y)^{* *}=\left(x^{*} \odot y^{*}\right)^{*}=x \boxplus y$. We prove that $(n x)^{* *}=n x$, for $n \geq 2$, by mathematical induction on $n$. For $n=2,(2 x)^{* *}=2 x$ is true from $(x \boxplus y)^{* *}=x \boxplus y$, for $x=y$. If we suppose that $(n x)^{* *}=n x$, for some $n \geq 2$, then $[(n+1) x]^{* *}=[x \boxplus(n x)]^{* *}=$ $x \boxplus(n x)=(n+1) x$.

$\left(c_{13}\right) . x \boxplus\left(y^{* *} \wedge z^{* *}\right)=x^{*} \rightarrow\left(y^{* *} \wedge z^{* *}\right) \stackrel{\left(c_{5}\right)}{=} x^{*} \rightarrow\left(y^{* *} \wedge z^{* *}\right) \stackrel{\left(c_{3}\right)}{=}\left(x^{*} \rightarrow y^{* *}\right) \wedge\left(x^{*} \rightarrow\right.$ $\left.z^{* *}\right)=(x \boxplus y) \wedge(x \boxplus z)$.

$\left(c_{14}\right)$. We have $\left(x^{* *} \wedge y^{* *}\right) \boxplus\left(x^{* *} \wedge z^{* *}\right)=\left(x^{* *} \wedge y^{* *}\right)^{*} \rightarrow\left(x^{* *} \wedge z^{* *}\right)^{* *} \stackrel{\left(c_{5}\right)}{=}\left(x^{* *} \wedge y^{* *}\right)^{*} \rightarrow$ $\left(x^{* *} \wedge z^{* *}\right) \stackrel{\left(c_{3}\right)}{=}$

$\left[\left(x^{* *} \wedge y^{* *}\right)^{*} \rightarrow x^{* *}\right] \wedge\left[\left(x^{* *} \wedge y^{* *}\right)^{*} \rightarrow z^{* *}\right]=\left[x^{*} \rightarrow\left(x^{* *} \wedge y^{* *}\right)^{* *}\right] \wedge\left[z^{*} \rightarrow\left(x^{* *} \wedge y^{* *}\right)^{* *}\right]=$ $\left[x^{*} \rightarrow\left(x^{* *} \wedge y^{* *}\right)\right] \wedge\left[z^{*} \rightarrow\left(x^{* *} \wedge y^{* *}\right)\right]=\left[x^{*} \rightarrow\left(x^{* *} \wedge y^{* *}\right)\right] \wedge\left[z^{*} \rightarrow x^{* *}\right] \wedge\left[z^{*} \rightarrow y^{* *}\right]$. Since $x \leq x^{*} \rightarrow\left(x^{* *} \wedge y^{* *}\right)$ and $x \leq x^{* *} \leq z^{*} \rightarrow x^{* *}$ we deduce that $x \leq\left[x^{*} \rightarrow\left(x^{* *} \wedge y^{* *}\right)\right] \wedge\left[z^{*} \rightarrow\right.$ $\left.x^{* *}\right]$, so $x \wedge(y \boxplus z)=x \wedge\left(y^{*} \rightarrow z^{* *}\right) \leq\left[x^{*} \rightarrow\left(x^{* *} \wedge y^{* *}\right)\right] \wedge\left[z^{*} \rightarrow x^{* *}\right] \wedge\left[y^{*} \rightarrow z^{* *}\right]$ $=\left(x^{* *} \wedge y^{* *}\right) \boxplus\left(x^{* *} \wedge z^{* *}\right)$.

$\left(c_{15}\right)$. First we show that $x \wedge(n y) \leq n\left(x^{* *} \wedge y^{* *}\right)$ by mathematical induction on $n$. For $n=2$, by $\left(c_{14}\right), x \wedge(2 y)=2\left(x^{* *} \wedge y^{* *}\right)$. If we suppose that $x \wedge n y \leq n\left(x^{* *} \wedge y^{* *}\right)$, then $x \wedge(n+1) y=x \wedge(y \boxplus n y) \stackrel{\left(c_{14}\right)}{\leq}\left(x^{* *} \wedge y^{* *}\right) \boxplus\left(x^{* *} \wedge(n y)^{* *}\right) \stackrel{\left(c_{12}\right)}{=}\left(x^{* *} \wedge y^{* *}\right) \boxplus\left(x^{* *} \wedge(n y)\right) \leq$ $\left(x^{* *} \wedge y^{* *}\right) \boxplus\left[n\left(x^{* *} \wedge y^{* *}\right)\right]=(n+1)\left(x^{* *} \wedge y^{* *}\right)$. Also, we have $(m x) \wedge(n y) \leq n\left[(m x)^{* *} \wedge\right.$ $\left.y^{* *}\right] \stackrel{\left(c_{12}\right)}{=} n\left[(m x) \wedge y^{* *}\right] \leq(m n)\left(x^{* *} \wedge y^{* *}\right)$.

Theorem 3.1. Let $I$ be a nonempty subset of $L$. Then $I$ is an i-ideal of $L$ iff $[0 \in I$ and for every $x, y \in L$ if $x, x \rightsquigarrow y \in I$, then $y \in I]$.

Proof. First, let $I \subseteq L$ be an i-ideal. Clearly $0 \in I$ and we consider $x, y \in L$ such that $x, x \rightsquigarrow y \in I$. Then $x \boxplus(x \rightsquigarrow y) \in I$, that is, $x^{*} \rightarrow(x \rightsquigarrow y)^{* *}=x^{*} \rightarrow(x \rightsquigarrow y)^{* *}=$ $x^{*} \rightarrow\left(x^{*} \odot y\right)^{* *}=x^{*} \rightarrow\left(x^{*} \odot y^{* *}\right) \in I$. Since $y \leq x^{*} \rightarrow\left(x^{*} \odot y^{* *}\right)$ we deduce that $y \in I$. Conversely, we consider $x, y \in L$ such that $x \leq y$ and $y \in I$. Since $y^{*} \odot x \leq x^{*} \odot x=0$, we obtain $y, y \rightsquigarrow x \in I$, so, $x \in I$. Let now $x, y \in I$. Since $y, y \rightsquigarrow y^{*}=0 \in I$ we get $y^{* *} \in I$, so from $x \rightsquigarrow(x \boxplus y)=x^{*} \odot\left(x^{*} \rightarrow y^{* *}\right) \leq y^{* *} \in I$ we deduce that $x \rightsquigarrow(x \boxplus y) \in I$. From $x, x \rightsquigarrow(x \boxplus y) \in I$ we obtain $x \boxplus y \in I$, hence $I$ is an i-ideal.

Theorem 3.1 is a kind of dual modus ponens for i-ideals.

We recall that if $A$ is an MV algebra and $I$ is an ideal of $A$ then the binary relation $x \sim_{I} y$ iff $x^{*} \odot y, x \odot y^{*} \in I$, for $x, y \in A$, is a congruence relation on $A$. Moreover, $I=\left\{x \in L: x \sim_{I} 0\right\}$. Also the assignment $I \rightsquigarrow \sim_{I}$ is a bijection from the set $\mathbf{I}_{i}(\mathbf{A})$ onto the set of congruences on $A$, see [4]. 
In [11], for a residuated lattice $L$ and an i-ideal $I$ of $L$ is defined the congruence relation $x \approx_{I} y$ iff $(x \rightarrow y)^{*},(y \rightarrow x)^{*} \in I$.

In the following for an i-ideal $I$ of a residuated lattice $L$ we study the relationships between the binary relations $\sim_{I}$ and $\approx_{I}$ on residuated lattices and we find classes of residuated lattices for which the relation $\sim_{I}$ (defined for MV algebras) is a congruence relation.

So, let $I$ be an i-ideal of a residuated lattice $L$ and we consider the binary relations $x \sim_{I} y$ iff $x^{*} \odot y, x \odot y^{*} \in I$ iff $x \rightsquigarrow y, y \rightsquigarrow x \in I$ and $x \approx_{I} y$ iff $(x \rightarrow y)^{*}$ and $(y \rightarrow x)^{*} \in I$.

Proposition 3.3. For $x, y \in L$ the following hold: $\left[x \sim_{I} y\right.$ iff $\left.x^{*} \approx_{I} y^{*}\right]$ and $\left[x \approx_{I} y\right.$ implies $x \sim I y]$

Proof. Clearly, $x \sim_{I} y$ iff $x^{*} \odot y, x \odot y^{*} \in I$ iff $\left(x^{*} \odot y\right)^{* *},\left(x \odot y^{*}\right)^{* *} \in I$ iff $\left(x^{*} \rightarrow y^{*}\right)^{*}$, $\left(y^{*} \rightarrow x^{*}\right)^{*} \in I$ iff $x^{*} \approx_{I} y^{*}$. Now we suppose that $x \approx_{I} y$. Since $\approx_{I}$ is a congruence relation on $L$ we deduce that $x^{*} \approx_{I} y^{*}$, so $x \sim_{I} y$.

Remark 3.3. In general, if $L$ is a residuated lattice, $x, y \in L$ and $I \in \mathbf{I}_{i}(\mathbf{L})$ then $x \sim_{I} y$ does not imply $x \approx_{I} y$. For example, if we consider the residuated lattice from Example 3.1. For $I=\{0\}$ we have $a \sim_{I} b$ since $a^{*} \odot b=a \odot b^{*}=0 \in I$, but $(b \rightarrow a)^{*}=n \notin I$, so $a$ is not in relation $\approx_{I}$ with $b$.

Theorem 3.2. For a residuated lattice $L$ and $I \in \mathbf{I}_{i}(\mathbf{L})$, the relation $\sim_{I}$ is an equivalence relation on $L$ compatible with $\odot, \vee,{ }^{*},{ }^{* *}$ and $\boxplus$.

Proof. Clearly, the relation $\sim_{I}$ is reflexive and symmetric. To prove the transitivity of $\sim_{I}$ let $x, y, z \in L$ such that $x \sim_{I} y$ and $y \sim_{I} z$. Since $\left(x^{*} \odot y\right)^{*} \odot\left(x^{*} \odot z\right)=\left(x^{*} \rightarrow\right.$ $\left.y^{*}\right) \odot x^{*} \odot z \leq y^{*} \odot z \in I$, we deduce that $\left(x^{*} \odot y\right) \rightsquigarrow\left(x^{*} \odot z\right) \in I$, hence, by Theorem 3.1, $x^{*} \odot z \in I$. Similarly, $x \odot z^{*} \in I$, hence $x \sim_{I} z$, that is, $\sim_{I}$ is transitive. Now we prove the compatibility of $\sim_{I}$ with $\odot, \vee,{ }^{*},{ }^{* *}$ and $\boxplus$. Let $a, x, y \in L$ such that $x \sim_{I} y$. Since $(a \odot x)^{*} \odot(a \odot y)=\left(a \rightarrow x^{*}\right) \odot a \odot y \leq x^{*} \odot y \in I$ we deduce that $(a \odot x)^{*} \odot(a \odot y) \in I$. Similarly, $(a \odot x) \odot(a \odot y)^{*} \in I$, so $(a \odot x) \sim_{I}(a \odot y)$. Also we have $(a \vee x)^{*} \odot(a \vee y)=$ $\left(a^{*} \wedge x^{*}\right) \odot(a \vee y)=\left[\left(a^{*} \wedge x^{*}\right) \odot a\right] \vee\left[\left(a^{*} \wedge x^{*}\right) \odot y\right]$. Since $a^{*} \wedge x^{*} \leq a^{*}$ we deduce that $\left(a^{*} \wedge x^{*}\right) \odot a \leq a^{*} \odot a=0$, so $(a \vee x)^{*} \odot(a \vee y)=\left(a^{*} \wedge x^{*}\right) \odot y \leq x^{*} \odot y \in I$, hence $(a \vee x)^{*} \odot(a \vee y) \in I$. Similarly, $(a \vee x) \odot(a \vee y)^{*} \in I$, so $(a \vee x) \sim_{I}(a \vee y)$. From Proposition 3.3, $x \sim_{I} y \Leftrightarrow x^{*} \approx_{I} y^{*} \Rightarrow x^{*} \sim_{I} y^{*}$ and as an immediate consequence, $x \sim_{I} y \Rightarrow x^{* *} \sim_{I} y^{* *}$.

To prove the compatibility of $\sim_{I}$ with $\boxplus$, we use the compatibility of $\sim_{I}$ with $\odot$ and * We deduce that $x \sim_{I} y$ implies $x^{*} \sim_{I} y^{*}$, so $\left(x^{*} \odot a^{*}\right) \sim_{I}\left(y^{*} \odot a^{*}\right)$. Thus, $\left(x^{*} \odot a^{*}\right)^{*} \sim_{I}$ $\left(y^{*} \odot a^{*}\right)^{*}$ and $(x \boxplus a) \sim_{I}(y \boxplus a)$.

Remark 3.4. $x \sim_{I} x^{* *}$, for every $x \in L$. Indeed, $x^{*} \odot x^{* *}=x \odot\left(x^{* *}\right)^{*}=0 \in I$.

Let $L$ be a residuated lattice, $I \in \mathbf{I}_{i}(\mathbf{L})$ and $x \in L$. We denote by $x / I$ the equivalence class of $x$ modulo $\sim_{I}$ and by $L / I=\{x / I: x \in L\}$ the factor set. For $x, y \in L$ we define on $L / I$ the unary operation $(x / I)^{*}=x^{*} / I$ and the binary operation $x / I \boxplus y / I=(x \boxplus y) / I$. Following Theorem 3.2 these operations are well defined. Clearly, $0 / I=\left\{x \in L: x \sim_{I}\right.$ $0\}=\{x \in L: x \in I\}=I$ and $1 / I=\left\{x \in L: x \sim_{I} 1\right\}=\left\{x \in L: x^{*} \in I\right\}$. 
Example 3.2. ([6]) Let $L=\{0, b, c, d, 1\}$ with $0<b, c<d<1$ and $b, c$ incomparable. $L$ becomes a residuated lattice relative to the following operations:

\begin{tabular}{|c|c|c|c|c|c|c|c|c|c|c|c|}
\hline$\rightarrow$ & 0 & $b$ & $c$ & $d$ & 1 & $\odot$ & 0 & $b$ & $c$ & $d$ & 1 \\
\hline 0 & 1 & 1 & 1 & 1 & 1 & 0 & 0 & 0 & 0 & 0 & 0 \\
\hline$b$ & $d$ & 1 & $d$ & 1 & 1 & $b$ & 0 & 0 & 0 & 0 & $b$ \\
\hline$c$ & $d$ & $d$ & 1 & 1 & 1 & $c$ & 0 & 0 & 0 & 0 & $c$ \\
\hline$d$ & $d$ & $d$ & $d$ & 1 & 1 & $d$ & 0 & 0 & 0 & 0 & $d$ \\
\hline 1 & 0 & $b$ & $c$ & $d$ & 1 & 1 & 0 & $b$ & $c$ & $d$ & 1 \\
\hline
\end{tabular}

Remark 3.5. The relation $\sim_{I}$ is not compatible with $\wedge$ and $\rightarrow$. Indeed, if we consider the residuated lattice $L$ from Example 3.2, then for $I=\{0\} \in \mathbf{I}_{i}(\mathbf{L})$ we have $b \sim_{I} d$ since $b^{*} \odot d=b \odot d^{*}=0$, but $(b \wedge c) \nsim_{I}(d \wedge c)$ since $0 \nsim_{I} c\left(0^{*} \odot c=c \notin I\right)$. Also, $(b \rightarrow b) \nsim_{I}(d \rightarrow b)$ since $1 \nsim_{I} d$ (because $\left.d^{*} \odot 1=d \notin I\right)$.

Theorem 3.3. If $I$ is an $i$-ideal of a residuated lattice $L$, then $(L / I, \boxplus, I)$ is a commutative monoid and for every $x \in L, x / I=(x / I)^{* *}$ and $1 / I \boxplus x / I=1 / I$.

Proof. Using $\left(c_{6}\right)$ we deduce that $\boxplus$ is associative and commutative on $L / I$. Clearly, for $x \in L, x / I \boxplus 0 / I=(x \boxplus 0) / I \stackrel{\left(c_{6}\right)}{=} x^{* *} / I=x / I$, since $x \sim_{I} x^{* *}$, from Remark 3.4. We conclude that $(L / I, \boxplus, 0 / I)$ is a commutative monoid. Also, $1 / I \boxplus x / I=(1 \boxplus x) / I=1 / I$, and by Remark 3.4, $x \sim_{I} x^{* *}$ so $x / I=x^{* *} / I=(x / I)^{* *}$.

Remark 3.6. If we consider the residuated lattice $L$ from Example 3.1 and $I=\{0\} \in \mathbf{I}_{i}(\mathbf{L})$, then $\left(n^{*} \boxplus a\right)^{*} \boxplus a=d$ and $\left(a^{*} \boxplus n\right)^{*} \boxplus n=1$. We remark that $\left(n^{*} \boxplus a\right)^{*} \boxplus a \nsim_{I}\left(a^{*} \boxplus n\right)^{*} \boxplus n$, since $1 \odot d^{*}=n \notin I$.

We recall that an $M V$ algebra (see [3]) is an algebra $\left(M, \oplus,{ }^{*}, 0\right)$ of type $(2,1,0)$ such that : $(M, \oplus, 0)$ is a commutative monoid; $x^{* *}=x, 1 \oplus x=1$, (where $1=0^{*}$ ) and $\left(x^{*} \oplus y\right)^{*} \oplus y=\left(y^{*} \oplus x\right)^{*} \oplus x$, for every $x, y \in M$.

Corollary 3.1. If $L$ a divisible residuated lattice and $I \in \mathbf{I}_{i}(\mathbf{L})$, then $\left(L / I, \boxplus,{ }^{*}, I\right)$ is an $M V$ algebra.

Proof. For $x, y \in L$ we have $\left(x^{*} \boxplus y\right)^{*} \boxplus y=\left(x^{* *} \odot y^{*}\right)^{* *} \boxplus y=\left(x^{* *} \odot y^{*}\right) \boxplus y=\left[\left(x^{* *} \odot\right.\right.$ $\left.\left.y^{*}\right)^{*} \odot y^{*}\right]^{*}=\left[y^{*} \odot\left(y^{*} \rightarrow x^{*}\right)\right]^{*}=\left(y^{*} \wedge x^{*}\right)^{*}=\left(y^{*} \boxplus x\right)^{*} \boxplus x$.

We deduce that $\left((x / I)^{*} \boxplus(y / I)\right)^{*} \boxplus(y / I)=\left((y / I)^{*} \boxplus(x / I)\right)^{*} \boxplus(x / I)$. From Theorem 3.3 we conclude that $\left(L / I, \boxplus,^{*}, I\right)$ is an MV algebra.

Lemma 3.1. Let $L$ be a residuated lattice, $I \in \mathbf{I}_{i}(\mathbf{L})$ and $x, y \in L$. Then the following are equivalent:

(i) $x \sim_{I} y$

(ii) $\left(x^{* *} \rightarrow a^{* *}\right) \sim_{I}\left(y^{* *} \rightarrow a^{* *}\right)$, for every $a \in L$;

(iii) $\left(a^{* *} \rightarrow x^{* *}\right) \sim_{I}\left(a^{* *} \rightarrow y^{* *}\right)$, for every $a \in L$;

(iv) $\left(x^{* *} \wedge a^{* *}\right) \sim_{I}\left(y^{* *} \wedge a^{* *}\right)$, for every $a \in L$.

Proof. $(i) \Rightarrow(i i),($ iii $),(i v)$. Using the compatibility of $\sim_{I}$ with $\odot, \vee,{ }^{*}$ and ${ }^{* *}$.

$($ ii $) \Rightarrow(i)$. For $a=x, 1 \sim_{I} y^{* *} \rightarrow x^{* *}$, so $\left(y^{* *} \rightarrow x^{* *}\right)^{*}=\left(y^{* *} \odot x^{*}\right)^{* *}=y^{* *} \odot x^{*} \in I$, thus, $y \odot x^{*} \in I$. Similarly, for $a=y$ we obtain $x \odot y^{*} \in I$, so $x \sim_{I} y$.

$($ iii $) \Rightarrow(i)$. Similarly with $(i i) \Rightarrow(i)$.

$(i v) \Rightarrow(i)$. For $a=1$, we deduce $x^{* *} \sim_{I} y^{* *}$. Using Remark 3.4, $x \sim_{I} y$.

Theorem 3.4. Let $L$ be a residuated lattice and $I \in \mathbf{I}_{i}(\mathbf{L})$.

(i) If $L$ is divisible then $\sim_{I}$ is a congruence relation on $L$;

(ii) If $L$ is a Girard monoid then $\sim_{I}$ is a congruence relation on $L$; 
(iii) If $L$ is a De Morgan residuated lattice with property that $(x \rightarrow y)^{* *}=x^{* *} \rightarrow y^{* *}$, for every $x, y \in L$, then $\sim_{I}$ is a congruence relation on $L$.

Proof. $(i)$. We recall that if $L$ is a divisible residuated lattice, then for every $x, y \in L,(x \wedge$ $y)^{* *}=x^{* *} \wedge y^{* *}$ and $(x \rightarrow y)^{* *}=x^{* *} \rightarrow y^{* *}$, see [1].

To prove the compatibility of $\sim_{I}$ with $\wedge$ and $\rightarrow$, let $x, y, a \in L$ such that $x \sim_{I} y$. Since $a \sim_{I} a^{* *}$ using Lemma 3.1, we have the following:

$$
\begin{gathered}
x \rightarrow a \sim_{I}(x \rightarrow a)^{* *}=x^{* *} \rightarrow a^{* *} \sim_{I} y^{* *} \rightarrow a^{* *}=(y \rightarrow a)^{* *} \sim_{I} y \rightarrow a, \\
a \rightarrow x \sim_{I}(a \rightarrow x)^{* *}=a^{* *} \rightarrow x^{* *} \sim_{I} a^{* *} \rightarrow y^{* *}=(a \rightarrow y)^{* *} \sim_{I} a \rightarrow y, \\
x \wedge a \sim_{I}(x \wedge a)^{* *}=x^{* *} \wedge a^{* *} \sim_{I} y^{* *} \wedge a^{* *}=(y \wedge a)^{* *} \sim_{I} y \wedge a .
\end{gathered}
$$

From Theorem 3.2 we conclude that $\sim_{I}$ is a congruence relation on $L$.

(ii). From Theorem 3.2 and Lemma 3.1 since $z=z^{* *}$, for every $z \in L$.

(iii). Similarly with $(i)$, using the fact that if $L$ is a De Morgan residuated lattice then $(x \wedge y)^{* *}=x^{* *} \wedge y^{* *}$, for every $x, y \in L$.

\section{PRIME I-IDEALS IN RESidUATED LATTICES}

In this section we give new characterizations for prime i-ideals in residuated lattices. The notion of prime i-ideal in residuated lattices generalizes the concept of prime ideal in MV algebras.

Proposition 4.4. Let $L$ be a residuated lattice, $x, y \in L$ and $I \in \mathbf{I}_{i}(\mathbf{L})$. Then $I(x \wedge y) \subseteq$ $I(x) \cap I(y) \subseteq I\left(x^{* *} \wedge y^{* *}\right)$

Proof. Clearly, $I \subseteq I(x) \cap I(y)$. Since $x \wedge y \leq x, y$ we deduce that $I(x \wedge y) \subseteq I(x), I(y)$ so $I(x \wedge y) \subseteq I(x) \cap I(y)$. Let now $z \in I(x) \cap I(y)$. Then, $z \leq i \boxplus(m x)$ and $z \leq j \boxplus(n y)$ with $i, j \in I$ and $m, n \geq 1$. If denote $k=i \boxplus j \in I$, then $z \leq k \boxplus m x$ and $z \leq k \boxplus n y$. If $m, n \geq 2$ we deduce successively $z \leq(k \boxplus m x) \wedge(k \boxplus n y)=\left[k^{*} \rightarrow(m x)^{* *}\right] \wedge\left[k^{*} \rightarrow(n y)^{* *}\right]=\left[k^{*} \rightarrow\right.$ $(m x)] \wedge\left[k^{*} \rightarrow(n y)\right] \stackrel{\left(c_{3}\right)}{=} k^{*} \rightarrow[(m x) \wedge(n y)]$

$\stackrel{\left(c_{15}\right)}{\leq} k^{*} \rightarrow\left[m n\left(x^{* *} \wedge y^{* *}\right)\right] \stackrel{\left(c_{12}\right)}{=} k^{*} \rightarrow\left[m n\left(x^{* *} \wedge y^{* *}\right)\right]^{* *}=k \boxplus\left[m n\left(x^{* *} \wedge y^{* *}\right)\right]$, hence $z \in I\left(x^{* *} \wedge y^{* *}\right)$. Similarly for $m=n=1$ and for $m=1, n \geq 2$ (or conversely). We deduce that $I(x) \cap I(y) \subseteq I\left(x^{* *} \wedge y^{* *}\right)$.

Definition 4.3. ([13]) Let $L$ be a residuated lattice. An i-ideal $I \in \mathbf{I}_{i}(\mathbf{L})$ is called prime if $I \neq L$ and $I$ is a prime element in the lattice $\left(\mathbf{I}_{i}(\mathbf{L}), \subseteq\right)$, that is, if $J, K \in \mathbf{I}_{i}(\mathbf{L})$ and $J \cap K \subseteq I$, then $J \subseteq I$ or $K \subseteq I$.

Because $\left(\mathbf{I}_{i}(\mathbf{L}), \subseteq\right)$ is a distributive lattice, then meet-irreducible and meet-prime elements are the same. So, $I$ is a prime element in the lattice $\left(\mathbf{I}_{i}(\mathbf{L}), \subseteq\right)$, that is, if $J, K$ $\in \mathbf{I}_{i}(\mathbf{L})$ and $J \cap K=I$, then $J=I$ or $K=I$.

Proposition 4.5. ([13]) Let $L$ be a residuated lattice and $I \in \mathbf{I}_{i}(\mathbf{L})$. Then:

(i). I is prime iff $x^{* *} \wedge y^{* *} \in I$ implies $x \in I$ or $y \in I$;

(ii) If $L$ is a De Morgan residuated lattice, $I$ is prime iff $x \wedge y \in I$ implies $x \in I$ or $y \in I$.

Proposition 4.6. Let $L$ be a residuated lattice and $I$ be a proper $i$-ideal of $L$ such that for every $x, y \in L, x^{*} \rightsquigarrow y^{*} \in I$ or $y^{*} \rightsquigarrow x^{*} \in I$. Then I is prime.

Proof. Let $x, y \in L$ such that $x^{* *} \wedge y^{* *} \in I$ and we suppose that $x^{*} \rightsquigarrow y^{*} \in I$. It follows that $\left(x^{* *} \odot y^{*}\right) \boxplus\left(x^{* *} \wedge y^{* *}\right) \stackrel{\left(c_{13}\right)}{=}\left[\left(x^{* *} \odot y^{*}\right) \boxplus y\right] \wedge\left[\left(x^{* *} \odot y^{*}\right) \boxplus x\right] \in I$. But $x \leq x^{* *}$, so $x \odot\left(x^{* *} \rightarrow y^{* *}\right) \leq x \odot\left(x \rightarrow y^{* *}\right) \leq y^{* *}$. Thus, $x \odot\left(x^{*} \boxplus y\right) \leq y^{* *} \Leftrightarrow x \leq\left(x^{* *} \odot y^{*}\right) \boxplus y$. Since $x \leq\left(x^{* *} \odot y^{*}\right) \boxplus x$, we deduce that $x \leq\left[\left(x^{* *} \odot y^{*}\right) \boxplus y\right] \wedge\left[\left(x^{* *} \odot y^{*}\right) \boxplus x\right]$. We get $x \in I$. Similarly, if $y^{*} \rightsquigarrow x^{*} \in I$ we obtain $y \in I$, so $I$ is prime. 
Example 4.3. ([6]) Let $L=\{0, a, b, c, 1\}$ with $0<a, b<c<1$ and $a, b$ incomparable. $L$ becomes a residuated lattice with the following operations:

\begin{tabular}{c|ccccc}
$\rightarrow$ & 0 & $a$ & $b$ & $c$ & 1 \\
\hline 0 & 1 & 1 & 1 & 1 & 1 \\
$a$ & $b$ & 1 & $b$ & 1 & 1 \\
$b$ & $a$ & $a$ & 1 & 1 & 1 \\
$c$ & 0 & $a$ & $b$ & 1 & 1 \\
1 & 0 & $a$ & $b$ & $c$ & 1
\end{tabular}

\begin{tabular}{c|ccccc}
$\odot$ & 0 & $a$ & $b$ & $c$ & 1 \\
\hline 0 & 0 & 0 & 0 & 0 & 0 \\
$a$ & 0 & $a$ & 0 & $a$ & $a$ \\
$b$ & 0 & 0 & $b$ & $b$ & $b$ \\
$c$ & 0 & $a$ & $b$ & $c$ & $c$ \\
1 & 0 & $a$ & $b$ & $c$ & 1
\end{tabular}.

It is immediate that $\mathbf{I}_{i}(\mathbf{L})=\{\{0\},\{0, a\},\{0, b\}, L\}$ and $\{0, a\},\{0, b\}$ are prime ideals of $L$. Also, we remark that $I=\{0\}$ is not prime since $a^{* *} \wedge b^{* *}=0 \in I$ but $a, b \notin I$.

Theorem 4.5. Let $L$ be an MTL algebra and $I \in \mathbf{I}_{i}(\mathbf{L})$. The following are equivalent:

(i) $I$ is a prime $i$-ideal of $L$;

(ii) $x^{*} \rightsquigarrow y^{*} \in I$ or $y^{*} \rightsquigarrow x^{*} \in I$, for every $x, y \in L$.

Proof. $($ ii $) \Rightarrow($ i). From Proposition 4.6.

$(i) \Rightarrow(i i)$. We have $\left(x^{*} \rightsquigarrow y^{*}\right) \wedge\left(y^{*} \rightsquigarrow x^{*}\right)=\left(x^{* *} \odot y^{*}\right) \wedge\left(y^{* *} \odot x^{*}\right)=\left(x^{* *} \odot y^{*}\right)^{* *} \wedge$ $\left(y^{* *} \odot x^{*}\right)^{* *}=\left(x^{* *} \rightarrow y^{* *}\right)^{*} \wedge\left(y^{* *} \rightarrow x^{* *}\right)^{*}=\left[\left(x^{* *} \rightarrow y^{* *}\right) \vee\left(y^{* *} \rightarrow x^{* *}\right)\right]^{*}=1^{*}=0$. Since MTL algebras are De Morgan residuated lattices, from Proposition 4.5, (ii), we deduce that $x^{*} \rightsquigarrow y^{*} \in I$ or $y^{*} \rightsquigarrow x^{*} \in I$.

Theorem 4.6. (Prime i-ideal theorem) Let $L$ be a residuated lattice. If $I \in \mathbf{I}_{i}(\mathbf{L})$ and $F$ is a filter in the lattice $(L, \wedge, \vee, 0,1)$ such that $I \cap F=\varnothing$, then there is a prime $i$-ideal $P$ of $L$ such that $I \subseteq P$ and $P \cap F=\varnothing$.

Proof. Let $\mathcal{I}_{I}=\left\{I^{\prime} \in \mathbf{I}_{i}(\mathbf{L}): I \subseteq I^{\prime}\right.$ and $\left.I^{\prime} \cap F=\varnothing\right\}$. Using Zorn's lemma we show that $\mathcal{I}_{I}$ has a maximal element $P$. Suppose by contrary that $P$ is not a prime i-ideal, that is, there are $a, b \in L$ such that $a^{* *} \wedge b^{* *} \in P$, but $a \notin P$ and $b \notin P$. By the maximality of $P$ we deduce that $P(a), P(b) \notin \mathcal{I}_{I}$, hence $P(a) \cap F \neq \varnothing$ and $P(b) \cap F \neq \varnothing$, that is, there are $p_{1} \in$ $P(a) \cap F$ and $p_{2} \in P(b) \cap F$. By Proposition 3.1, $p_{1} \leq f \boxplus(m a)$ and $p_{2} \leq g \boxplus(n b)$, with $f, g \in P$ and $m, n \geq 1$.

If $m, n \geq 2$, then $p_{1} \wedge p_{2} \leq(f \boxplus m a) \wedge(g \boxplus n b) \stackrel{\left(c_{14}\right)}{\leq}\left(f^{* *} \wedge g^{* *}\right) \boxplus\left(f^{* *} \wedge(n b)^{* *}\right) \boxplus\left((m a)^{* *} \wedge\right.$ $\left.g^{* *}\right) \boxplus\left((m a)^{* *} \wedge(n b)^{* *}\right) \stackrel{\left(c_{12}\right)}{=}\left(f^{* *} \wedge g^{* *}\right) \boxplus\left(f^{* *} \wedge(n b)\right) \boxplus\left((m a) \wedge g^{* *}\right) \boxplus((m a) \wedge(n b)) \stackrel{\left(c_{15}\right)}{\leq}$ $\left(f^{* *} \wedge g^{* *}\right) \boxplus\left(f^{* *} \wedge(n b)\right) \boxplus\left((m a) \wedge g^{* *}\right) \boxplus(m n)\left(a^{* *} \wedge b^{* *}\right)$.

Since $f^{* *} \wedge g^{* *}, f^{* *} \wedge(n b),(m a) \wedge g^{* *},(m n)\left(a^{* *} \wedge b^{* *}\right) \in P$ we deduce that $p_{1} \wedge p_{2} \in P \cap F$, hence $P \cap F \neq \varnothing$, a contradiction. Thus, $P$ is a prime. Similarly if $m=1$ or $n=1$.

Remark 4.7. Using Theorem 4.6, we remark that any proper i-ideal of a residuated lattice can be extended to a prime i-ideal.

Lemma 4.2. If $L$ is a De Morgan residuated lattice and $P$ is a prime $i$-ideal of $L$, then $L \backslash P$ is a filter in the lattice $(L, \wedge, \vee, 0,1)$.

Proof. Indeed, since $P$ is proper, $1 \in L \backslash P$. If $a \leq b$ and $a \in L \backslash P$, then $b \in L \backslash P$, since $P$ is an i-ideal of $L$. If $a, b \in L \backslash P$, then $a \wedge b \in L \backslash P$, since $P$ is prime.

Remark 4.8. In a residuated lattice $L$, for a prime i-ideal $P$ of $L, L \backslash P$ is not necessary a filter in the lattice $(L, \wedge, \vee, 0,1)$. Indeed, if we consider the residuated lattice $L=\{0, b, c, d, 1\}$ from Example 3.2 thus, $I=\{0\}$ is a prime i-ideal of $L$, but $L \backslash I=\{b, c, d, 1\}$ is not a filter in the lattice $(L, \wedge, \vee, 0,1)$, because $b \wedge c=0 \notin L \backslash I$. 
Example 4.4. ([6]) Let $L=\{0, n, a, b, c, d, m, 1\}$ with $0<n<a, b<c<m<1$ and $a, b$ are incomparable, $b<c, d<m<1$ and also $c$, $d$ are incomparable. $L$ becomes a residuated lattice relative to the following operations:

\begin{tabular}{c|cccccccc}
$\rightarrow$ & 0 & $n$ & $a$ & $b$ & $c$ & $d$ & $m$ & 1 \\
\hline 0 & 1 & 1 & 1 & 1 & 1 & 1 & 1 & 1 \\
$n$ & $m$ & 1 & 1 & 1 & 1 & 1 & 1 & 1 \\
$a$ & $d$ & $d$ & 1 & $d$ & 1 & $d$ & 1 & 1 \\
$b$ & $c$ & $c$ & $c$ & 1 & 1 & 1 & 1 & 1 \\
$c$ & $b$ & $b$ & $c$ & $d$ & 1 & $d$ & 1 & 1 \\
$d$ & $a$ & $a$ & $a$ & $c$ & $c$ & 1 & 1 & 1 \\
$m$ & $n$ & $n$ & $a$ & $b$ & $c$ & $d$ & 1 & 1 \\
1 & 0 & $n$ & $a$ & $b$ & $c$ & $d$ & $m$ & 1
\end{tabular}

\begin{tabular}{c|cccccccc}
$\odot$ & 0 & $n$ & $a$ & $b$ & $c$ & $d$ & $m$ & 1 \\
\hline 0 & 0 & 0 & 0 & 0 & 0 & 0 & 0 & 0 \\
$n$ & 0 & 0 & 0 & 0 & 0 & 0 & 0 & $n$ \\
$a$ & 0 & 0 & $a$ & 0 & $a$ & 0 & $a$ & $a$ \\
$b$ & 0 & 0 & 0 & 0 & 0 & $b$ & $b$ & $b$ \\
$c$ & 0 & 0 & $a$ & 0 & $a$ & $b$ & $c$ & $c$ \\
$d$ & 0 & 0 & 0 & $b$ & $b$ & $d$ & $d$ & $d$ \\
$m$ & 0 & 0 & $a$ & $b$ & $c$ & $d$ & $m$ & $m$ \\
1 & 0 & $n$ & $a$ & $b$ & $c$ & $d$ & $m$ & 1
\end{tabular}.

It is easy to see that $x^{* *}=x$, for every $x \in L$.

Remark 4.9. In a residuated lattice, a proper i-ideal that contains a prime i-ideal is not necessarily prime. Indeed, if we consider the residuated lattice $L=\{0, n, a, b, c, d, m, 1\}$ from Example 4.4, then $I_{1}=\{0\}, I_{2}=\{0, n\}, I_{3}=\{0, n, a\}, I_{4}=\{0, n, b, d\}$ are i-ideals. Moreover, $I_{1}, I_{3}$ and $I_{4}$ are prime i-ideals of $L$ and $I_{2}$ is not a prime i-ideal of $L$ (because $a^{* *} \wedge b^{* *}=n \in I_{2}$ but $\left.a, b \notin I_{2}\right)$. We remark that $I_{1}$ is a prime, $I_{1} \subseteq I_{2}$, but $I_{2}$ is not a prime i-ideal of $L$.

Remark 4.10. If $L$ is a BL algebra, then every proper i-ideal of $L$ that contains a prime i-ideal is prime (see [9], Proposition 5.17).

Remark 4.11. In a residuated lattice, the set of proper i-ideals including a prime i-ideal is not a chain. Indeed, if we consider the residuated lattice $L=\{0, n, a, b, c, d, m, 1\}$ from Example 4.4, then the set of proper i-ideals of $L$ including the prime i-ideal $I_{1}=\{0\}$ is not a chain because $I_{3} \nsubseteq I_{4}$ and $I_{4} \nsubseteq I_{3}$.

Remark 4.12. If $L$ is a BL algebra, then for a prime i-ideal $P$ of $L$ the set $\mathcal{I}_{P}=\left\{I \in \mathbf{I}_{i}(\mathbf{L})\right.$ : $P \subseteq I$ and $I \neq L\}$ is totally ordered by inclusion, see [13].

We recall that an i-ideal $I$ of a residuated lattice $L$ is called maximal (see [13]) if it is proper and it is not contained in any other proper i-ideal of $L$. Obviously, in a residuated lattice, every proper i-ideal can be extended to a maximal i-ideal and every maximal iideal is prime.

Example 4.5. If we consider the residuated lattice $L$ from Example 3.1 it is easy to see that the prime i-ideals are $\{0\},\{0, n\}$ and the only maximal i-ideal is $\{0, n\}$.

In a residuated lattice $L$, we denote the intersection of maximal i-ideals by $\operatorname{Rad}_{I d}(L)$. Obviously that $\operatorname{Rad}_{I d}(L) \in \mathbf{I}_{i}(\mathbf{L})$.

Proposition 4.7. In a residuated lattice $L, B(L) \cap \operatorname{Rad}_{I d}(L)=\{0\}$, where $B(L)$ is the set of all complemented elements of $L$.

Proof. We recall that if $e \in B(L)$ then $e \vee e^{*}=1, e \wedge e^{*}=0$ and $e=e^{* *}$. Obviously, $0 \in B(L) \cap \operatorname{Rad}_{I d}(L)$. Let $e \in B(L), e \neq 0$. By Theorem 4.6, there is a prime i-ideal $P$ of $L$ such that $e \notin P$. We have $0=e \wedge e^{*}=e^{* *} \wedge\left(e^{*}\right)^{* *} \in P$, so $e^{*} \in P$ (since $P$ is prime and $\left.e=e^{* *} \notin P\right)$. Also, there is a maximal i-ideal $M$ such that $P \subseteq M$. It follows that $e^{*} \in M$, so $e \notin M$, because if $e \in M$, then $e \boxplus e^{*} \stackrel{\left(c_{6}\right)}{=} 1 \in M$, a contradiction. Thus, $e \notin \operatorname{Rad}_{I d}(L)$. 
As a generalization of the case of BL algebras (see [9]) we investigate the relationship between ideals and deductive systems in residuated lattices.

Let $L$ be a residuated lattice.

Definition 4.4. ([14]) A subset $D$ of $L$ is called deductive system if : $1 \in D$ and [If $x, x \rightarrow$ $y \in D$, then $y \in D]$.

An equivalent definition for a deductive system is ([14]):

$\left(d s_{1}\right)$ If $x \leq y$ and $x \in D$, then $y \in D$;

$\left(d s_{2}\right)$ If $x, y \in D$, then $x \odot y \in D$.

Following this equivalence, a deductive system of $L$ is also called an implicative filter. We denote by $D s(L)$ the set of deductive systems of $L . D \in D s(L)$ is called prime if $D \neq L$ and for $x, y \in L$, if $x \vee y \in D$ then $x \in D$ or $y \in D$.

We recall that for any subset $X$ of a residuated lattice $L$ we denote by $X^{*}=\left\{x^{*}: x \in\right.$ $X\}$ and by $N(X)=\left\{x \in L: x^{*} \in X\right\}$ the set of complement elements (with respect to $X$ ).

As a generalization of the case of $B L$-algebras (see [9]) we have:

Theorem 4.7. Let $L$ be a residuated lattice, $I \in \mathbf{I}_{i}(\mathbf{L})$ and $D \in D s(L)$. Then

(i) $N(I) \in D s(L)$ and $I^{*} \subseteq N(I)$;

(ii) $N(D)$ is the $i$-ideal of $L$ generated by $D^{*}$;

(iii) $I=N(N(I))$;

(iv) $D \subseteq N(N(D))$ and $N(D)=N(N(N(D)))$.

Proof. $(i)$. Since $I \in \mathbf{I}_{i}(\mathbf{L})$ and $0 \in I$ we deduce that $1 \in N(I)$. Let $x, y \in N(I)$. Then $x^{*}, y^{*} \in I, x^{*} \boxplus y^{*}=x^{* *} \rightarrow y^{*}=y \rightarrow x^{*}=(y \odot x)^{*} \in I$, thus $x \odot y \in N(I)$. Now let $x, y \in L$ such that $x \leq y$ and $x \in N(I)$. Then $x^{*} \in I$. Since $y^{*} \leq x^{*}, x^{*} \in I$, we deduce that $y^{*} \in I$, thus $y \in N(I)$. We conclude that $N(I) \in D s(L)$.

Obviously, $I^{*} \subseteq N(I)$, since for any $x \in L, x \in I$ iff $x^{* *} \in I$.

(ii). Since $D \in D s(L)$ and $1 \in D$ we deduce that $0 \in N(D)$.

Let $x, y \in N(D)$. Then $x^{*}, y^{*} \in D$. Since $D \in D s(L)$, then $x^{*} \odot y^{*} \in D$. But $x^{*} \odot y^{*} \leq$ $\left(x^{*} \odot y^{*}\right)^{* *}$, so $(x \boxplus y)^{*} \in D$ and $x \boxplus y \in N(D)$. Now let $x, y \in L$ such that $x \leq y$ and $y \in N(D)$. Then $y^{*} \in D$. Since $y^{*} \leq x^{*}, y^{*} \in D$ we deduce that $x^{*} \in D$, thus $x \in N(D)$. We conclude that $N(D) \in \mathbf{I}_{i}(\mathbf{L})$.

To prove that $N(D)$ is generated by $D^{*}$, we prove first that $D^{*} \subseteq N(D)$.

Let $x^{*} \in D^{*}$, with $x \in D$. Then $x^{* *} \in D$ because $x \leq x^{* *}$ and $D$ is a deductive system. Hence $x^{*} \in N(D)$ so $D^{*} \subseteq N(D)$. Finally, let $J$ be an i-ideal containing $D^{*}$ and let $x \in$ $N(D)$. Then $x^{*} \in D$, so $x^{* *} \in D^{*} \subseteq J$. Hence $x^{* *} \in J$ so $x \in J$. Thus $N(D)=\left(D^{*}\right]$.

(iii). Since $I$ is an i-ideal of $L$, then $N(N(I))=\left\{x \in L: x^{* *} \in I\right\}=\{x \in L: x \in I\}=I$.

(iv). Let $x \in D$. Since $x \leq x^{* *}$, then $x^{* *} \in D$, so $x \in N(N(D))$ and $D \subseteq N(N(D))$. Since $D \in D s(L)$, from $(i i), N(D) \in \mathbf{I}_{i}(\mathbf{L})$. Using $(i i i)$, for $I=N(D)$, we deduce that $N(D)=N(N(N(D)))$.

Theorem 4.8. Let $L$ be a residuated lattice, $I \in \mathbf{I}_{i}(\mathbf{L})$ and $D \in D s(L)$. Then

(i) If $I$ is a prime $i$-ideal of $L$, then $N(I)$ is a prime deductive system of $L$;

(ii) If $L$ is a De Morgan residuated lattice and $D$ is a prime deductive system of $L$, then $N(D)$ is a prime $i$-ideal of $L$.

Proof. (i). Using Theorem 4.7, $(i), N(I) \in D s(L)$. Let $x, y \in L$ such that $x \vee y \in N(I)$. Then $(x \vee y)^{*}=x^{*} \wedge y^{*}=\left(x^{*}\right)^{* *} \wedge\left(y^{*}\right)^{* *} \in I$. Since $I$ is a prime $\mathrm{i}$-ideal we deduce that $x^{*} \in I$ or $y^{*} \in I$, so $x \in N(I)$ or $y \in N(I)$. Thus, $N(I)$ is a prime deductive system of $L$.

(ii). Using Theorem 4.7, $(i i), N(D) \in \mathbf{I}_{i}(\mathbf{L})$. Let $x, y \in L$ such that $x \wedge y \in N(D)$. Then $(x \wedge y)^{*}=x^{*} \vee y^{*} \in D$. Since $D$ is a prime deductive system of $L$ we deduce that $x^{*} \in D$ or $y^{*} \in D$, so $x \in N(D)$ or $y \in N(D)$. Hence $N(D)$ is a prime i-ideal of $L$. 
Using Theorems 4.7 and 4.8, we conclude that:

Corollary 4.2. Let $L$ be a De Morgan residuated lattice and $I \in \mathbf{I}_{i}(\mathbf{L})$. Then I a prime $i$-ideal of $L$ iff $N(I)$ is a prime deductive system of $L$.

\section{REFERENCES}

[1] Buşneag, D., Piciu, D. and Paralescu, I., Divisibile and semi-divisibile residuated lattices, Ann St. Univ. A. I. Cuza, Iaşi, Matem, LXI (f.2) (2015), 287-318

[2] Buşneag, D., Piciu, D. and Holdon, L., Some properties of ideals in Stonean Residuated Lattices, J. Mult.-Valued Logic Soft Comput., 24 (2015), No. 5-6, 529-546

[3] Cignoli, R., D'Ottaviano, I. M. L. and Mundici, D., Algebraic Foundations of many-valued Reasoning, Trends in Logic-Studia Logica Library 7, Dordrecht: Kluwer Academic Publishers, 2000

[4] Chang, C. C., Algebraic analysis of many-valued logic, Trans. Amer. Math. Soc., 88 (1958), 467-490

[5] Dilworth, R. P., Non-commutative residuated lattices, Trans. Amer. Math. Soc., 46 (1939), 426-444

[6] Iorgulescu, A., Classes of BCK algebras-Part III , Preprint Series of The Institute of Mathematics of the Romanian Academy, Preprint No. 3 (2004), 1-37

[7] Kengne, P. C., Koguep, B. B., Akume, D. and Lele, C., L-fuzzy ideals of residuated lattices, Discuss. Math. Gen. Algebra Appl., 39 (2019), No. 2, 181-201

[8] Krull, W., Axiomatische Begründung der allgemeinen Ideal theorie, Sitzungsberichte der physikalisch medizinischen Societäd der Erlangen, 56 (1924), 47-63

[9] Lele, C. and Nganou, J. B., MV-algebras derived from ideals in BL-algebras, Fuzzy Sets and Systems, 218 (2013), 103-113

[10] Lele, C. and Nganou, J. B., Pseudo-addition and fuzzy ideals in BL-algebras, Ann. Fuzzy Math. and Inform., 8 (2014), 193-207

[11] Liu, Y., Qin, Y., Qin, X. and Xu, Y., Ideals and fuzzy ideals in residuated lattices, Int. J. Math. Learn \& Cyber., 8 (2017), 239-253

[12] Pavelka, J., On fuzzy logic II. Enriched residuated lattices and semantics of propositional calculi, Z. Math. Logik Grundlagen Math., 25 (1979), No. 2, 119-134

[13] Piciu, D., Busneag, D. and Istrata, M., Pure ideals and stable topology for residuated lattices, submitted to International Journal of Fuzzy Systems

[14] Turunen, E., Mathematics Behind Fuzzy Logic, Physica-Verlag, 1999

[15] Ward, M. and Dilworth, R. P., Residuated lattices, Trans. Amer. Math. Soc., 45 (1939), No. 3, 335-354

DEPARTMENT OF MATHEMATICS

UNIVERSITY OF CRAIOVA, FACULTY OF SCIENCE

A. I. Cuza Street, 13, 200585, CRAiova, Romania

Email address: busneag@central .ucv.ro

Email address: piciudanamarina@yahoo.com

Email address: ancadinaucv@yahoo.com 